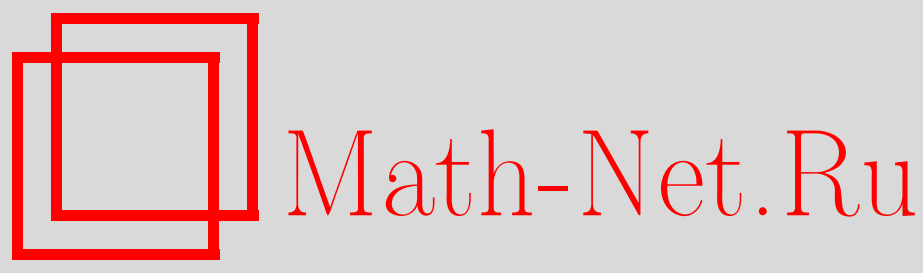

М. В. Комарова, Д. М. Краснов, М. Ю. Налимов, Бозеконденсация: критическая размерность вязкости, развитая турбулентность, ТМФ, 2011, том 169, номер 1, 89-99

DOI: https://doi.org/10.4213/tmf6711

Использование Общероссийского математического портала Math-Net.Ru подразумевает, что вы прочитали и согласны с пользовательским соглашением http://www.mathnet.ru/rus/agreement

Параметры загрузки:

IP: 54.224 .60 .19

26 апреля 2023 г., 03:22:07

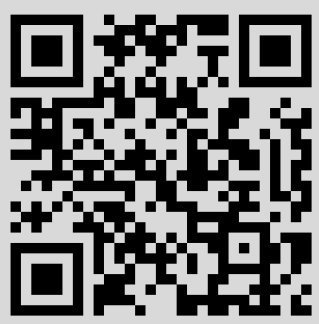




\title{
БОЗЕ-КОНДЕНСАЦИЯ: КРИТИЧЕСКАЯ РАЗМЕРНОСТЬ ВЯЗКОСТИ, РАЗВИТАЯ ТУРБУЛЕНТНОСТЬ
}

\begin{abstract}
Предложена модель для исследования взаимного влияния критических флуктуаций в окрестности критической точки фазового перехода в сверхтекучее состояние и флуктуаций скорости в режиме развитой турбулентности. Показано наличие в зависимости от турбулентной накачки энергии двух различных режимов: турбулентного и равновесного. Показано, что в турбулентном режиме обыкновенное критическое поведение может разрушиться. В равновесном режиме вязкость является инфракрасно несущественным параметром. Обосновано предположение, что критическая размерность вязкости в этом режиме определяется достаточно точно известными в настоящий момент критическими индексами статической модели критического поведения.
\end{abstract}

Ключевые слова: бозе-конденсация, развитая турбулентность, ренормализационная группа, стохастическая динамика, критическое поведение.

\section{1. ВВЕДЕНИЕ}

Бозе-конденсация - фазовый переход второго рода в квантовой системе. В основе традиционного описания лежит статистическая сумма газа с локальным взаимодействием типа “плотность-плотность" [1]:

$$
\Sigma=C \int D \psi^{+} D \psi e^{-S_{\beta}\left(\psi^{+}, \psi\right)} .
$$

Поля $\psi^{+}$и $\psi$ обладают свойством обобщенной комплексной сопряженности [2]: $\psi^{+}(t, \mathbf{x})=\psi^{*}(-t, \mathbf{x}), C-$ нормировочная константа, которая при определенных правилах доопределения в функциональном интеграле не зависит ни от спектра энергии, ни от температуры. Поля заданы на интервале $t \in[0, \beta=1 / k T]$ с периодическими граничными условиями $\left.\psi(\mathbf{x}, t)\right|_{t=0}=\left.\psi(\mathbf{x}, t)\right|_{t=\beta}$. Действие $S_{\beta}$ в формализме

${ }^{*}$ Санкт-Петербургский государственный университет, Санкт-Петербург, Россия.

E-mail: frommarina@mail.ru,dmitriy.m.krasnov@gmail.com, mikhail.nalimov@pobox.spbu.ru 
температурных функций Грина имеет вид

$$
\begin{aligned}
S_{\beta}= & \int_{0}^{\beta} d t \int d \mathbf{x} \psi^{+}(\mathbf{x}, t)\left(\partial_{t}+\hat{\epsilon}\right) \psi(\mathbf{x}, t)+ \\
& +\int_{0}^{\beta} d t \int d \mathbf{x} \int d \mathbf{x}^{\prime} \psi^{+}(\mathbf{x}, t) \psi(\mathbf{x}, t) V\left(\mathbf{x}-\mathbf{x}^{\prime}\right) \psi^{+}\left(\mathbf{x}^{\prime}, t\right) \psi\left(\mathbf{x}^{\prime}, t\right), \quad \hat{\epsilon}=\frac{\hat{p}^{2}}{2 m}-\mu .
\end{aligned}
$$

Данное действие определяет пропагатор свободной теории в виде

$$
G\left(t^{\prime}, t\right)=e^{-\epsilon\left(t^{\prime}-t\right)}\left[\Theta\left(t^{\prime}-t\right)+\frac{1}{e^{\beta \epsilon}-1}\right],
$$

который в критической области $(\mu, p \rightarrow 0)$ превращается в $G\left(t^{\prime}, t\right) \approx(\beta \epsilon)^{-1}$. Теперь поля $\psi$ и $\psi^{+}$становятся независимыми от $t$, а действие эффективной теории превращается в стандартное действие статической теории $\phi^{4}(n=2)$, которое и описывает статические свойства критического поведения в окрестности точки фазового перехода.

К сожалению, аналогичный строгий формализм для описания динамики критических флуктуаций не развит. При решении этой задачи вместо точных микроскопических квантовых уравнений используются феноменологические модели, основанные на стохастических уравнениях Ланжевена, описывающих динамику классических переменных - полей параметра порядка и температуры (модели F и Е стохастической динамики [3], [4]). Методом ренормализационной группы критическая динамика в данных моделях была рассмотрена в двухпетлевом приближении в работе [5]. Гидродинамические моды оказываются инфракрасно (ИК) несущественны в критической области и отсутствуют в моделях Е, F.

Однако наиболее известным примером фазового перехода бозе-конденсации является переход жидкого гелия в сверхтекучее состояние, при котором обращается в нуль вязкость сверхтекучей компоненты. Стремление к нулю вязкости приводит к возрастанию числа Рейнольдса $\operatorname{Re}=L v / \nu(L-$ характерный размер системы, $v$ - характерная скорость, $\nu$ - кинематическая вязкость). При нестационарных граничных условиях этой ситуации соответствует появление в системе развитой турбулентности (хотя следует признать, что обычно явление сверхтекучести наблюдается в тонких капиллярах, где число Рейнольдса может быть ограничено толщиной капилляра). Поэтому нами была рассмотрена проблема взаимодействия критических флуктуаций и явления развитой турбулентности. Настоящая статья в значительной степени посвящена скейлинговой размерности вязкости.

Следует пояснить, что мы будем понимать под скейлинговой размерностью. Мы рассматриваем не только критический, но и турбулентный режим. Обычно при описании динамики критического поведения кинетическому коэффициенту приписывают нулевую критическую размерность, при этом вклад его аномальной размерности относят к динамическому индексу $z$ [4]. В рассматриваемой системе имеется три разных кинетических коэффициента (включая кинематическую вязкость). Аномальная размерность одного из этих коэффициентов может быть отнесена к индексу $z$, при этом скейлинговая размерность остальных коэффициентов оказывается нетривиальной. В теории развитой турбулентности традиционно вводят нетривиальную скейлинговую размерность вязкости [4]. Поэтому мы сохраним традиционное для 
критической динамики определение индекса $z$ через кинетические коэффициенты моделей $\mathrm{F}$ и $\mathrm{E}$, а под скейлинговой размерностью вязкости будем понимать по сути ее аномальную размерность, имея в виду, что в корреляционные функции вязкость входит, в частности, в виде безразмерной комбинации $p^{2} \bar{\nu} t$, где $p-$ волновое число, $t$ - время, а $\bar{\nu}$ - инвариантная вязкость, скейлинговое поведение которой мы и обсуждаем.

Для описания критического поведения и развитой турбулентности в работе используется метод квантово-полевой ренормализационной группы (РГ) (см., например, [4]). В рамках РГ-формализма обоснована и исследована модель, в которой гидродинамические моды становятся существенными в логарифмической теории. Применяемый подход основан на двойном разложении по параметрам $\epsilon=4-d$ $(d-$ размерность пространства) и $\delta$ - отклонению степени накачки турбулентной энергии от логарифмического значения. Отметим, что данный подход был развит в работах [6], [7] и использован в [8] для учета влияния гидродинамических мод в модели А критической динамики.

За основу описания взяты уравнения стохастической динамики, приводящие на больших временах к равновесному распределению (при подходящей функции накачки турбулентной энергии).

В однопетлевом приближении вычислены РГ-функции (коэффициенты уравнения РГ). Обосновано существование в зависимости от накачки турбулентной энергии двух принципиально различных скейлинговых режимов: равновесного и турбулентного. В турбулентном режиме (аналогично [8]) показано, что развитая турбулентность, возможно, разрушает обыкновенное критическое поведение. Физически такая картина объясняется тем, что турбулентные вихри ограничивают рост корреляционного радиуса критических флуктуаций, а также тем, что сверхтекучесть проявляется при скоростях жидкости, не превышающих некоторое критическое значение.

Показано, что в турбулентном режиме скейлинговая размерность вязкости такая же, как и в обыкновенной теории развитой турбулентности. В равновесном режиме вязкость является ИК-несущественным параметром, как и предполагалось ранее в посвященных динамике бозе-конденсации работах [3], [5], при этом скейлинговая размерность вязкости определяется поправочными индексами $\omega$ ИК-устойчивой фиксированной точки уравнения РГ. Для вычисления этой размерности необходимо установить, какой именно фиксированной точкой уравнения РГ описывается интересующий нас режим. K сожалению, данная задача не была решена даже в двухпетлевом приближении в более простых моделях F и Е [5]. Проблема заключается в том, что соответствующие индексы имеют малые значения, поэтому их знак не удается определить из первых порядков разложения. Необходимы многопетлевые вычисления и последующее пересуммирование рядов теории возмущений или качественные экспериментальные результаты (что и используется при описании жидкого гелия).

Тем не менее нами обнаружена возможная связь скейлингового индекса вязкости с известными критическими индексами статической модели С, которые установлены достаточно надежно как по результатам пятипетлевых (шестипетлевых в трехмерном пространстве) вычислений [4], так и экспериментально. 
Структура статьи такова: в разделе 2 рассмотрены модели $\mathrm{F}$ и $\mathrm{E}$ стохастической динамики. Приведены результаты однопетлевых расчетов функций $\beta$ и $\gamma$ в данных моделях. Выведены стохастические уравнения модели F с включением гидродинамических мод, сформулирован новый, основанный на двойном разложении, подход, в котором вязкость становится маргинальной в логарифмической модели. В разделе 3 рассматривается ренормировка предложенной модели и приведены результаты однопетлевых вычислений РГ-функций в теории с гидродинамическими модами. В разделе 4 проведен анализ полученных уравнений, установлены возможные ИК-устойчивые скейлинговые режимы. В разделе 5 кратко суммированы основные выводы, следующие из приведенного анализа.

\section{2. МОДЕЛИ КРИТИЧЕСКОЙ ДИНАМИКИ}

Критическая динамика традиционно описывается стохастическими уравнениями Ланжевена, приводящими при больших временах к равновесному распределению Гиббса. Равновесное распределение имеет вид $\rho \sim e^{S_{\mathrm{st}}}$, где стационарное действие выбирается в виде [3], [4]

$$
S_{\mathrm{st}}=-\partial \psi^{+} \partial \psi-\frac{g_{1}}{6}\left(\psi^{+} \psi\right)^{2}+g_{2} m \psi^{+} \psi-\frac{1}{2} m^{2}-\frac{1}{2} v^{2}+m h .
$$

Здесь поля $\psi, \psi^{+}$- параметр порядка, $m$ - некоторая линейная комбинация внутренней энергии и плотности, ответственная за флуктуации температуры, $v$ - поле скорости, которое предполагается поперечным, что возможно в дозвуковом диапазоне (аналогично модели Н и работе [8]). Следует отметить, что, в отличие от работ [3], [4], где ИК-несущественность гидродинамических мод была установлена до написания уравнений движения, мы включили поле скорости в статическое действие (1) в виде максвелловского распределения по аналогии с моделью Н критической динамики [3], [4].

Теперь несложно построить динамические уравнения Ланжевена для рассматриваемой модели. Критерием выбора уравнений в данной форме является то, что они приводят к равновесному распределению на больших временах:

$$
\begin{gathered}
\partial_{t} \psi+\partial_{i}\left(v_{i} \psi\right)=\lambda(1+i b)\left[\partial^{2} \psi-\frac{g_{1}\left(\psi^{+} \psi\right) \psi}{3}+g_{2} m \psi\right]+ \\
+i \lambda g_{3} \psi\left[g_{2} \psi^{+} \psi-m+h\right]+f_{\psi^{+}}
\end{gathered}
$$

уравнение на поле $\psi^{+}$получается комплексным сопряжением предыдущего уравнения,

$$
\begin{aligned}
\partial_{t} m+ & \partial_{i}\left(v_{i} m\right)=-\lambda u \partial^{2}\left[g_{2} \psi^{+} \psi-m+h\right]+i \lambda g_{3}\left[\psi^{+} \partial^{2} \psi-\psi \partial^{2} \psi^{+}\right]+f_{m}, \\
\partial_{t} v= & \nu \partial^{2} v-\partial_{i}\left(v_{i} v\right)-\psi^{+} \partial\left[\partial^{2} \psi-\frac{g_{1}}{3}\left(\psi^{+} \psi\right) \psi+g_{2} m \psi\right]- \\
& \quad \psi \partial \partial\left[\partial^{2} \psi^{+}-\frac{g_{1}}{3}\left(\psi^{+} \psi\right) \psi^{+}+g_{2} m \psi^{+}\right]-m \partial\left[g_{2} \psi^{+} \psi-m+h\right]+f_{v} .
\end{aligned}
$$

Здесь и далее $\partial$ - дифференцирование по пространственной компоненте, $\partial^{2}$ - оператор Лапласа. Отметим, что уравнение (4) есть стохастическое уравнение Навье- 
Стокса, дополненное членами, необходимыми для существования равновесного статистического предела у предложенной модели.

Для случайных сил $f_{i}$ предполагаются гауссовы распределения с корреляторами:

$$
\begin{gathered}
\left\langle f_{\psi} f_{\psi^{+}}\right\rangle=D_{\psi}\left(p, t, t^{\prime}\right)=\lambda \delta\left(t-t^{\prime}\right), \quad\left\langle f_{m} f_{m}\right\rangle=D_{m}\left(p, t, t^{\prime}\right)=\lambda u p^{2} \delta\left(t-t^{\prime}\right), \\
\left\langle f_{v} f_{v}\right\rangle=D_{v}\left(p, t, t^{\prime}\right)=\nu p^{2} \delta\left(t-t^{\prime}\right) .
\end{gathered}
$$

Спектр модели $\omega \sim p^{2}$ определяется уравнениями (2)-(4). В критической области $p \rightarrow 0, h \rightarrow 0$ и следует приписать следующие канонические размерности полям модели (см. [4]):

$$
\begin{array}{ccrl}
d[\psi]=\frac{d}{2}-1, & d\left[\psi^{\prime}\right]=\frac{d}{2}+1, & d[m]=\frac{d}{2}, \\
d\left[m^{\prime}\right]=\frac{d}{2}, & d[v]=1, & d\left[v^{\prime}\right]=\frac{d}{2},
\end{array}
$$

что и доказывает ИК-несущественность поля $v$.

Динамическое действие модели $\mathrm{F}$ в формализме Мартина-Сигиа-Розе [9] получается из уравнений (2) после отбрасывания ИК-несущественных членов:

$$
\begin{aligned}
S=2 \lambda & \left(\psi^{+}\right)^{\prime} \psi^{\prime}-\lambda u m^{\prime} \partial^{2} m^{\prime}+\left(\psi^{+}\right)^{\prime}\left\{-\partial_{t} \psi+\lambda(1+i b)\left[\partial^{2} \psi-\frac{g_{1}}{3}\left(\psi^{+} \psi\right) \psi+g_{2} m \psi\right]+\right. \\
& \left.+i \lambda g_{3} \psi\left[g_{2} \psi^{+} \psi-m+h\right]\right\}+\psi^{\prime}\left\{-\partial_{t} \psi^{+}+\lambda(1-i b)\left[\partial^{2} \psi^{+}-\right.\right. \\
& \left.\left.-\frac{g_{1}}{3}\left(\psi^{+} \psi\right) \psi^{+}+g_{2} m \psi^{+}\right]-i \lambda g_{3} \psi^{+}\left[g_{2} \psi^{+} \psi-m+h\right]\right\}+ \\
& +m^{\prime}\left\{-\partial_{t} m-\lambda u \partial^{2}\left[g_{2} \psi^{+} \psi-m+h\right]+i \lambda g_{3}\left[\psi^{+} \partial^{2} \psi-\psi \partial^{2} \psi^{+}\right]\right\} .
\end{aligned}
$$

Критическое поведение в модели F рассмотрено в статье [5] в двухпетлевом приближении в рамках $d=4-\epsilon$ разложения. Несмотря на то что действие содержит больше членов, чем общее количество полей и зарядов, данная теория мультипликативно-ренормируема [4]. Для строгого доказательства данного факта были использованы следующие общие соотношения: 1) существование равновесного статического предела; 2) по крайней мере одна производная во взаимодействии может быть переброшена интегрированием по частям на поле $m^{\prime}$, что понижает на единицу реальный индекс расходимости ампутированных графов с внешним хвостом $m^{\prime}$, поэтому, в частности, отсутствуют контрчлены вида $m^{\prime} \partial_{t} m$ и имеется связь $Z_{m}^{\prime} Z_{m}=1$ на константы ренормировки; 3) существует симметрия $\left\{\psi, \psi^{\prime}\right\} \rightarrow e^{i \alpha(t)}\left\{\psi, \psi^{\prime}\right\}$, $\left\{\psi^{+},\left(\psi^{+}\right)^{\prime}\right\} \rightarrow e^{-i \alpha(t)}\left\{\psi^{+},\left(\psi^{+}\right)^{\prime}\right\},\left\{m, m^{\prime}\right\} \rightarrow\left\{m, m^{\prime}\right\}$, откуда следует еще одна связь $Z_{\lambda} Z_{g_{3}}=Z_{m}$.

Далее мы приводим результаты однопетлевых вычислений РГ-функций зарядов, полей и кинетических коэффициентов модели F. Используя связь функций $\beta$ и $\gamma$ с константами ренормировки $Z_{i}$, в новых переменных $g_{1} \rightarrow g_{1} /\left(8 \pi^{2}\right), g_{i \neq 1} \rightarrow$ $g_{i \neq 1} / \sqrt{8 \pi^{2}}$ можно написать

$$
\begin{gathered}
\beta_{g_{1}}=g_{1}\left(-\epsilon-\gamma_{g_{1}}\right), \quad \beta_{g_{2}}=g_{2}\left(-\frac{\epsilon}{2}-\gamma_{g_{2}}\right), \quad \beta_{g_{3}}=g_{3}\left(-\frac{\epsilon}{2}-\gamma_{g_{3}}\right), \\
\beta_{u}=-u \gamma_{u}, \quad \beta_{b}=-b \gamma_{b},
\end{gathered}
$$


где $\gamma_{i}=\mu \partial_{\mu} \ln Z_{i}, \mu$ - ренормировочная масса,

$$
\begin{aligned}
\gamma_{g_{1}} & =-g_{1}\left(\frac{5}{3}-\frac{6 g_{2}^{4}}{g_{1}^{2}}\right)-\frac{1}{2} g_{2}\left(-\frac{20}{3} g_{2}+\frac{24 g_{2}^{2}}{g_{1}}\right), \quad \gamma_{g_{2}}=-\frac{2}{3} g_{1}+\frac{3}{2} g_{2}^{2}, \\
\gamma_{g_{3}} & =-\frac{2 b^{2} g_{2}^{2} u-b^{2} g_{2}^{2}-4 b g_{2} g_{3} u+g_{2}^{2} u^{2}-g_{2}^{2}+2 g_{3}^{2}+2 g_{3}^{2} u}{2\left((1+u)^{2}+b^{2}\right)}, \\
\gamma_{u} & =-\frac{g_{2}^{2} u^{3}+u^{2}\left(b^{2} g_{2}^{2}-2 b g_{2} g_{3}-3 g_{3}^{2}+g_{2}^{2}\right)-7 g_{3}^{2} u-4 g_{3}^{2} b^{2}-4 g_{3}^{2}}{u\left((u+1)^{2}+b^{2}\right)}, \\
\gamma_{b} & =-\frac{g_{2}^{2} u b\left(b^{2}+1\right)-2 g_{2} g_{3}(u+1)\left(b^{2}+1\right)+g_{3}^{2} b(u+2)}{b\left((u+1)^{2}+b^{2}\right)}, \\
\gamma_{m} & =-\frac{1}{2} g_{2}^{2}, \quad \gamma_{\left(\psi^{+}\right)^{\prime}}=\gamma_{\psi^{\prime}}^{*}=\frac{\left(g_{2}(1-i b)+i g_{3}\right)\left(g_{2}(1+i b)+u g_{2}\right)}{(1+u)^{2}}, \\
\gamma_{m^{\prime}} & =\frac{1}{2} g_{2}^{2}, \quad \gamma_{\psi^{+}}=\gamma_{\psi}=0, \\
\gamma_{\lambda} & =\frac{b^{2} g_{2}^{2} u-b^{2} g_{2}^{2}-2 b g_{2} g_{3} u-g_{2}^{2}-g_{2}^{2} u+g_{3}^{2} u+g_{3}^{2}}{(u+1)^{2}+b^{2}} .
\end{aligned}
$$

Отметим, что результаты для РГ-функций полей и кинетических коэффициентов достаточно трудно извлечь из работы [5]. Это связано с тем, что в модели имеется порядка 50 фиксированных точек, среди которых требуется определить ИК-устойчивую (с соответствующей положительно определенной матрицей $\omega$ ). Одним из основных результатов работы [5] следует признать установление сложности использования $\epsilon$-разложения при решении данной задачи. А именно, не только первый порядок разложения, но даже двухпетлевые вычисления (а это общепринятый ныне уровень аналитических вычислений в динамических моделях) оказываются недостаточными для определения ИК-устойчивой фиксированной точки уравнения РГ. Ответ на поставленный вопрос был получен в результате борелевского суммирования пяти порядков $\epsilon$-разложения в статической модели С [3], [4] и путем экспериментального измерения индекса теплоемкости $\alpha$ в жидком гелии. В результате принято, что ИК-устойчивые скейлинговые режимы описываются упрощением модели F - моделью Е критической динамики. Недостаточность результатов первых порядков $\epsilon$-разложения, по-видимому, и является причиной того, что в работе [5] приведены далеко не все результаты РГ-анализа, а ИК-устойчивый режим был выбран, по сути, априори.

Модель Е получается из рассмотренной выше, если положить $g_{2}=b=0$. Как показано в монографии [4], эта модель также мультипликативно ренормируема. Для доказательства данного факта, помимо вышеупомянутых свойств модели $\mathrm{F}$, используется дополнительная симметрия $\psi \rightarrow \psi^{+},\left\{m, m^{\prime}, h\right\} \rightarrow\left\{-m,-m^{\prime},-h\right\}$. Из существования статического предела в данном случае следует $Z_{m}=1$.

Результаты РГ-анализа в модели Е следующие: возможно существование двух критических режимов, причем двухпетлевой уровень вычислений также оказывается недостаточным для установления того, какой из них является ИК-устойчивым [4], [5].

Вернемся к проблеме определения критической размерности вязкости. Вслед за гидродинамическими модами вязкость оказывается ИК-несущественным параметром, и согласно канонической схеме РГ-анализа ее критическая размерность должна 
быть определена размерностью некоторого составного оператора, например $\nu v^{\prime} \Delta v^{\prime}$ или $\nu v^{\prime} \Delta v$. Но структура взаимодействия (6) такова, что оба эти оператора дают нулевой вклад в функции Грина моделей Е и F. Становится необходимым исследование ИК-поправок высоких порядков, однако практически в данный момент это нереально - ведь требуется еще и пересуммировать $\epsilon$-разложения для реальных значений $\epsilon$.

В настоящей статье предложена новая модель, а именно мы предлагаем вместо коррелятора скорости (5) использовать нелокальное представление

$$
D_{v}=g_{4} \nu^{3} p^{\epsilon-\delta} \delta\left(t-t^{\prime}\right)
$$

как это было выполнено ранее в работах [6], [7] и принято в теории развитой турбулентности (см. [4]). При $g_{4}=\nu^{-2}$ и $\epsilon-\delta=2$ это представление совпадает с (5).

Логарифмической теории теперь соответствует $\epsilon=\delta=0$, критические характеристики теории предполагается строить в виде двойного $(\delta, \epsilon)$-разложения. Физическими значениями являются точки $\delta=-1, \epsilon=1$ для описания равновесного режима и, как обычно, $\delta=4, \epsilon=1$ для режима развитой турбулентности в окрестности критической точки.

Вследствие физической определенности и относительной простоты в качестве основы рассмотрения мы выбрали модель Е. Действие модели Е с включением гидродинамических мод имеет вид

$$
\begin{aligned}
S= & 2 \lambda\left(\psi^{+}\right)^{\prime} \psi^{\prime}-\lambda u m^{\prime} \partial^{2} m^{\prime}+v^{\prime} D_{v} v^{\prime}+\left(\psi^{+}\right)^{\prime}\left\{-\partial_{t} \psi-\partial_{i}\left(v_{i} \psi\right)+\right. \\
& \left.+\lambda\left[\partial^{2} \psi-g_{1}\left(\psi^{+} \psi\right) \frac{\psi}{3}\right]+i \lambda g_{3} \psi[-m+h]\right\}+ \\
& +\psi^{\prime}\left\{-\partial_{t} \psi^{+}-\partial_{i}\left(v_{i} \psi^{+}\right)+\lambda\left[\partial^{2} \psi^{+}-g_{1}\left(\psi^{+} \psi\right) \frac{\psi^{+}}{3}\right]-\right. \\
& \left.-i \lambda g_{3} \psi^{+}[-m+h]\right\}+m^{\prime}\left\{-\partial_{t} m-\partial_{i}\left(v_{i} m\right)-\lambda u \partial^{2}[-m+h]+\right. \\
& \left.+i \lambda\left(g_{3}+\Delta g_{3}\right)\left[\psi^{+} \partial^{2} \psi-\psi \partial^{2} \psi^{+}\right]\right\}+v^{\prime}\left\{-\partial_{t} v+\nu \partial^{2} v-\partial_{i}\left(v_{i} v\right)\right\},
\end{aligned}
$$

в него не включены члены

$$
v^{\prime}\left\{-\psi^{+} \partial\left[\partial^{2} \psi-\frac{g_{1}}{3}\left(\psi^{+} \psi\right) \psi\right]-\psi \partial\left[\partial^{2} \psi^{+}-\frac{g_{1}}{3}\left(\psi^{+} \psi\right) \psi^{+}\right]-m \partial[-m+h]\right\},
$$

ИК-несущественные в предлагаемой схеме, как и в модели Е. Однако включенные теперь гидродинамические моды и вязкость оказываются маргинальными в логарифмической теории.

\section{3. РЕНОРМИРОВКА МОДЕЛИ}

При исследовании ренормируемости модели существенны следующие свойства: 1) следствием галилеевой инвариантности действия является тот факт, что члены действия, отличающиеся заменой $v \partial \rightarrow \partial_{t}$, ренормируются одинаково; 2) отсутствуют нелокальные контрчлены вида $v^{\prime} D_{v} v^{\prime}$ (как и в обычной теории развитой турбулентности), поэтому согласно соотношению $D_{v} \sim g_{4} \nu^{3}$ справедливо $Z_{g_{4}}=Z_{\nu}^{-3}$; 
3) безразмерная константа $\nu$ может быть представлена в виде $\nu=u_{1} \lambda$, новый заряд $u_{1}$ имеет свою константу ренормировки; 4) вид взаимодействия обеспечивает отсутствие генерационных вкладов в $Z_{g_{4}}$ и контрчленов вида $v^{\prime} \partial_{t} v, v^{\prime}(v \partial v)$ (как и в обычной теории развитой турбулентности); 5) во взаимодействии $\phi^{\prime} \partial_{i}\left(v_{i} \phi\right)$ производная может быть переброшена как на поле $\phi^{\prime}$, так и на $\phi$, что понижает реальный индекс расходимости соответствующих диаграмм; 6) константы ренормировки отличаются от констант ренормировки модели Е вкладами, обращающимися в нуль при нулевых $g_{4}$ и $\left.\Delta g_{3} ; 7\right)$ изменение коррелятора случайной силы $D_{v}$ привело к отсутствию равновесного предела теории - он должен восстановиться лишь при физическом равновесном значении параметра $\delta$.

Хотя построенная теория потеряла мультипликативность ренормировки, приведенные выше соображения показывают, что ее можно восстановить введением единственного нового заряда, связанного с новым членом действия $\Delta g_{3} m^{\prime}\left(\psi^{+} \partial^{2} \psi-\right.$ $\left.\psi \partial^{2} \psi^{+}\right)$. Как будет отмечено ниже, затравочным значением нового заряда, по-видимому, является $\Delta g_{3}=0$.

Приведем наши результаты однопетлевых вычислений в рассматриваемой модели (предполагается $\left.g_{4} \rightarrow g_{4} /\left(8 \pi^{2}\right)\right)$ :

$$
\begin{aligned}
& \gamma_{g_{1}}=-\frac{5}{3} g_{1}-\frac{3 g_{4} u_{1}^{2}}{8\left(1+u_{1}\right)}+\frac{\Delta g_{3} g_{3}}{(u+1)^{2}}+\frac{\Delta g_{3}^{2} g_{3}^{2}(u+2)}{g_{1} u(u+1)^{2}}- \\
& -\frac{\Delta g_{3} g_{3}^{3}(1+2 u)}{g_{1} u(u+1)^{2}}-\frac{g_{3} \Delta g_{3} u}{u+1}+\frac{g_{3} \Delta g_{3} u}{2(u+1)}-\frac{3 g_{3} \Delta g_{3}\left(u^{2}+3 u+2\right)}{2(u+1)^{2}}, \\
& \gamma_{g_{3}}=-\frac{g_{3}^{2}}{1+u}-g_{4}\left(-\frac{u_{1}^{2}}{8\left(1+u_{1}\right) u}+\frac{3 u_{1}^{2}}{8\left(1+u_{1}\right)}\right)- \\
& -g_{3} \Delta g_{3}\left(\frac{1}{u^{2}+3 u+2}-\frac{u^{2}+3 u+3}{(u+1)^{2}}+\frac{2}{u}\right)-\frac{2}{u} \Delta g_{3}^{2}, \\
& \gamma_{u}=-g_{3}^{2}\left(\frac{1}{1+u}-\frac{4}{u}\right)-\frac{3 g_{4} u_{1}^{2}}{1+u_{1}}\left(\frac{1}{8}-\frac{1}{4 u}\right)-g_{4} \Delta g_{3} \frac{u^{2}-4 u-4}{u(u+1)}, \\
& \gamma_{u_{1}}=\gamma_{\lambda}-\gamma_{\nu}, \quad \gamma_{\nu}=\frac{g_{4}}{8}, \quad \beta_{g_{4}}=g_{4}\left(-\delta+3 \gamma_{\nu}\right) \text {, } \\
& \gamma_{\Delta g_{3}}=\left(1+\frac{g_{3}}{\Delta g_{3}}\right) \frac{\left(1-2 u_{1}-2 u\right) 3 g_{4} u_{1}^{2}}{8\left(1+u_{1}\right)\left(u_{1}+u\right)}+\frac{g_{4} u_{1}^{2}}{8 u\left(1+u_{1}\right)}+\frac{3 g_{4} g_{3} u_{1}^{2}}{8\left(1+u_{1}\right) \Delta g_{3}}, \\
& \gamma_{m}=\frac{g_{4} u_{1}^{2}}{8\left(1+u_{1}\right) u}-\frac{2}{u}\left(g_{3} \Delta g_{3}+\Delta g_{3}^{2}\right) \text {, } \\
& \gamma_{\left(\psi^{+}\right)^{\prime}}=\gamma_{\psi^{\prime}}=-\frac{3 g_{4} u_{1}^{2}}{16\left(1+u_{1}\right)}-\frac{g_{3} \Delta g_{3} u}{2(u+1)}, \\
& \gamma_{m^{\prime}}=-\frac{g_{4} u_{1}^{2}}{8\left(1+u_{1}\right) u}+\frac{2}{u}\left(g_{3} \Delta g_{3}+\Delta g_{3}^{2}\right), \\
& \gamma_{\psi^{+}}=\gamma_{\psi}=\frac{3 g_{4} u_{1}^{2}}{16\left(1+u_{1}\right)}+\frac{g_{3} \Delta g_{3}\left(u^{2}+3 u+2\right)}{2(u+1)^{2}}, \\
& \gamma_{\lambda}=\frac{g_{3}}{u+1}+\frac{3 g_{4} u_{1}^{2}}{8\left(1+u_{1}\right)}+\frac{g_{3} \Delta g_{3} u}{u+1} \text {. }
\end{aligned}
$$




\section{4. СКЕЙЛИНГОВЫЕ РЕЖИМЫ МОДЕЛИ}

Из приведенных выше выражений видно, что существуют два принципиально разных скейлинговых режима относительно заряда $g_{4}$, зависящих от знака параметра $\delta$ :

1) режим равновесных флуктуаций, когда в фиксированной точке $g_{4 *}=0(\delta<0$, $\left.\gamma_{\nu *}=0\right)$

$2)$ турбулентный режим, когда $g_{4 *} \neq 0\left(\delta>0, \gamma_{\nu *}=\delta / 3>0\right)$.

Для аномальных размерностей здесь введено обозначение $\gamma \cdot{ }_{\cdot *} \equiv .\left(\left\{g_{i *}\right\},\left\{u_{i *}\right\}, \Delta g_{3 *}\right)$, символом * обозначены заряды в фиксированной точке. Отметим, что как существование, так и анализ устойчивости, по крайней мере равновесного режима относительно $\beta_{g 4}$, является пертурбативно точным. Эти режимы различаются скейлинговой размерностью вязкости.

Имеется еще одно точное соотношение $\beta_{u 1}=-u_{1}\left(\gamma_{\nu}-\gamma_{\lambda}\right)$, что приводит еще к одной альтернативе, не зависящей от рассматриваемого порядка теории возмущений. Если $u_{1 *} \neq 0$, то $\gamma_{\nu *}=\gamma_{\lambda *}$, а при $u_{1 *}=0$ возможны независимые значения $\gamma_{\nu *}$ и $\gamma_{\lambda *}$.

Рассмотрим равновесный режим. Если устойчивой фиксированной точке соответствует $u_{1 *} \neq 0$, то аномальные размерности $\gamma_{\nu *}=\gamma_{\lambda *}=0$. Если же устойчивой оказывается фиксированная точка $u_{1 *}=0$, то $\gamma_{\nu *}=0$, а для динамического критического индекса $z$ справедливы результаты моделей $\mathrm{F}, \mathrm{E}$ [4], [5]; критический индекс $z$ или равен $d / 2$, или имеет нетривиальное значение, которое еще надлежит определить путем пересуммирования результатов многопетлевых вычислений. Судя по всему, ни теоретического, ни экспериментального определения устойчивого скейлингового режима в этих моделях в настоящее время нет.

Таким образом, в равновесном режиме всегда $\gamma_{\nu *}=0$, поэтому в ведущем ИКприближении канонически безразмерная вязкость является константой. Но поскольку речь идет о переходе в сверхтекучее состояние, эта константа, по-видимому, равна нулю, и имеет смысл рассматривать ИК-поправочные члены к скейлинговым законам. В нашей схеме кинематическая вязкость содержит заряд $u_{1}$, матрица смешивания зарядов с $g_{4}$ может быть нетривиальной, поэтому скейлинговое поведение вязкости (а может быть, и $\lambda$ ) в критической области определяется поправочными индексами матрицы $\omega$. В этом случае скейлинговая размерность вязкости строго отрицательна, что вполне согласуется с фактом обращения вязкости в нуль при переходе в сверхтекучее состояние.

Если ИК-устойчивой фиксированной точкой теории оказывается точка с $\Delta g_{3 *}=0$ и $u_{1 *} \neq 0$, а адекватной моделью описания равновесных флуктуаций в квантовом бозе-газе все же является модель $\mathrm{F}$, то один из поправочных индексов теории можно считать известным. А именно, из многопетлевых вычислений в статической модели $\phi^{4}$ установлен один из поправочных индексов матрицы $\omega$, равный $-\alpha /(2 \nu)$. Отметим, что он очень мал, в частности существенно меньше, чем другой точно известный индекс матрицы $\omega$ - связанный с зарядом $g_{4}$ индекс, равный $-\delta$. Поэтому в данной ситуации можно с большой степенью уверенности утверждать, что "аномальная размерность" вязкости есть именно $\alpha /(2 \nu)$.

4 Теоретическая и математическая физика, т. 169, № 1, 2011 г. 
В турбулентном режиме скейлинговая размерность вязкости известна точно, $\gamma_{\nu *}=\delta / 3$. Размерности же основных полей и параметров существенно зависят от значения $u_{1 *}$ в устойчивой фиксированной точке. При $u_{1 *} \neq 0$ поля $\psi, \psi^{+}$, $\psi^{\prime},\left(\psi^{+}\right)^{\prime}$ и параметр $\lambda$ приобретают скейлинговые размерности, отличные от критических (по крайней мере, в теории возмущений). Если же устойчивой оказывается фиксированная точка $u_{1 *}=0$, то развитая турбулентность может сосуществовать с обыкновенным критическим поведением.

\section{5. ЗАКЛЮЧЕНИЕ}

Нами показано, что в равновесном режиме "аномальная размерность" вязкости отрицательна; как и предполагалось, вязкость является ИК-несущественным параметром, что неудивительно, ибо речь идет о переходе в сверхтекучее состояние. Не исключено, что эта размерность определяется известным индексом $\omega$ статической теории, но для точного ответа на этот вопрос необходимо уточнить исходя из строгой микроскопической теории феноменологическую модель. Действительно, хотя модель $\mathrm{F}$ и является более общей и, по-видимому, приводит к модели $\mathrm{E}$ в ИК-области, в принципе возможно, что именно модель Е исходно является правильной для описания динамики флуктуаций в окрестности точки бозе-конденсации, и приведенный индекс $\omega$ не дает вклада в скейлинговую размерность вязкости.

В турбулентном режиме скейлинговое поведение может (если $u_{1 *} \neq 0$ ) отличаться от критического, аномальные размерности даже основных полей теории могут не совпадать со статическими. Скейлинговая размерность вязкости известна точно и положительна, т.е. вязкость является ИК-существенным параметром. Данный результат не удивителен, так как повторяет результаты для других моделей (см., например, [8]). Можно заключить, что в данном случае размеры критической области ограничиваются турбулентными вихрями, которые разрушают критическое поведение.

Одно- и двухпетлевые вычисления оказываются недостаточными, чтобы выбрать ИК-устойчивую фиксированную точку уравнения РГ даже в статической модели С, не говоря уже о динамической модели F. Очевидно, то же самое справедливо и для рассматриваемой модели при реальных значениях параметров разложения (именно поэтому в работе даже не приводятся соответствующие результаты однопетлевого анализа). Более того, динамическое критическое поведение не определяется однозначно из мацубаровского формализма, поэтому возможна ситуация, когда физически значимыми оказываются не ИК-устойчивые фиксированные точки, а седловые точки, неустойчивые направления которых исключаются из физических соображений микроскопической теории. В качестве примера следует упомянуть критические режимы, связанные с $\Delta g_{3 *}=0$, ибо они диктуются существованием равновесного предела в динамических уравнениях.

Для более точного описания данного явления необходимы уточнение феноменологической модели и многопетлевые вычисления констант ренормировки с последующим пересуммированием.

Благодарности. Работа выполнена при поддержке РФФИ (грант №08-02-00125a). 


\section{Список литературы}

[1] А. А. Абрикосов, Л. П. Горьков, И.Е. Дзялошинский, Методы квантовой теории поля в статистической физике, Наука, М., 1998.

[2] А.Н. Васильев, Функиионалънъе методы в квантовой теории поля и статистике, Изд-во ЛГУ, Л., 1976.

[3] P. C. Hohenberg, B. I. Halperin, Rev. Mod. Phys., 49:3 (1977), 435-479.

[4] А.Н. Васильев, Квантовополевая ренормгруппа в теории критического поведения и стохастической динамике, Изд-во ПИЯФ, СПб., 1998.

[5] C. De Dominicis, L. Peliti, Phys. Rev. B, 18:1 (1978), 353-376.

[6] J. Honkonen, M. Yu. Nalimov, J. Phys. A, 22:6 (1989), 751-763.

[7] J. Honkonen, M. Yu. Nalimov, Z. Phys. B, 99:2 (1996), 297-303.

[8] N. V. Antonov, M. Hnatic, J. Honkonen, J. Phys. A, 39:25 (2006), 7867-7887, arXiv: cond-mat/0604434.

[9] P. C. Martin, E. D. Siggia, H. A. Rose, Phys. Rev. A, 8:1 (1973), 423-437. 